\title{
An In-Depth Review of the Evidence Linking Dietary Salt Intake and Progression of Chronic Kidney Disease
}

\author{
Charlotte Jones-Burton ${ }^{a}$ Shiraz I. Mishra ${ }^{b}$ Jeffrey C. Fink ${ }^{a}$ Jeanine Brown ${ }^{a}$ \\ Weyinshet Gossa ${ }^{a}$ George L. Bakris ${ }^{c}$ Matthew R. Weir ${ }^{a}$ \\ ${ }^{\mathrm{a}}$ Department of Medicine, Division of Nephrology, and ${ }^{\mathrm{b}}$ Department of Epidemiology and Preventive Medicine, \\ and Office of Policy and Planning, University of Maryland School of Medicine, Baltimore, Md., and ${ }^{\mathrm{C}}$ Department of \\ Preventive Medicine, Hypertension/Clinical Research Center, Rush University Medical Center, Chicago, III., USA
}

\section{Key Words}

Kidney disease $\cdot$ Dietary sodium $\cdot$ Salt intake $\cdot$ Proteinuria $\cdot$ Albuminuria $\cdot$ Glomerular filtration rate

\begin{abstract}
Background: Dietary salt has been debated for decades as having a potentially deleterious influence on human health. Objectives: To determine the quality of research and the relationship between dietary salt and markers for progression of kidney disease. Methods: Data sources included 7 electronic databases comprehensively searched for literature published between January 1, 1966, and August 31, 2004, and a manual search of bibliographies of relevant papers, and consultation with experts in the field. Differences between the paired reviewers were reconciled through consensus or by a content expert. Results: Sixteen studies met the inclusion-exclusion criteria and were identified for review; however, the study methodologies were extremely heterogeneous. Conclusions commonly cited in the studies include: variations in salt consumption are directly correlated with albuminuria, and an increase in salt consumption is associated with an acute increase in glomerular filtration rate, while a reduction in salt consumption may slow the rate of renal function loss. Conclusions: The available published information, while highly variable in methods and quality,
\end{abstract}

suggests that variations in dietary salt consumption directly influence albuminuria, with increasing salt intake associated with worsening albuminuria; however, results are inadequate and conflicting on the effects of dietary salt consumption on renal function, especially over a prolonged time. There was no evidence of a detrimental effect of reduced salt intake. On the other hand, there is consistent experimental evidence to link increased salt exposure with kidney tissue injury. On the basis of these data, we believe that dietary salt restriction should be considered in patients with chronic kidney disease.

Copyright $\odot 2006$ S. Karger AG, Basel

\section{Introduction}

Chronic kidney disease (CKD) is an important, widespread clinical problem which has multiple etiologies. Control of blood pressure, cholesterol, and glucose are important strategies to slow progression of CKD towards end-stage renal disease (ESRD). Pharmacological manipulation of the renin-angiotensin system has demonstrated efficacy in conjunction with good blood pressure control in reducing the likelihood of progression of kidney disease to end-stage kidney failure [1]. However, it is frequently difficult to control blood pressure and approach

Matthew R. Weir, MD

Division of Nephrology, University of Maryland School of Medicine

22 S. Greene St., Room N3W143

Baltimore, MD 21201 (USA)

Tel. +1 410328 5720, Fax +1 410328 5685, E-Mail mweir@medicine.umaryland.edu 
the goals necessary to minimize the risks for progression of kidney disease for multiple reasons including system/ physician level factors (i.e. late identification of CKD, late referral to a nephrologist), and patient level factors (i.e. non-compliance, both pharmacologic and dietary). Given that there are few pharmacologic agents to control CKD progression, behavior modifications can make an important contribution to CKD management, specifically dietary salt restriction.

The relationship between salt and blood pressure has been discussed in medical literature for decades and it has been shown that increased salt intake contributes to the prevalence of hypertension and proteinuria [2]. Less intensely studied are the direct nephrotoxic effects of sodium chloride. Salt ingestion has been linked to kidney disease via hemodynamic (e.g. increased introglomerular pressure) and non-hemodynamic mechanisms (e.g. increased oxidative stress), independent of blood pressure [3]. To date, there has been no synthesis of the existing evidence regarding the association between salt intake and the progression of CKD.

Therefore, we conducted an in-depth review of all available scientific literature, regardless of study design, on the relationship between dietary $\mathrm{NaCl}$ and markers for progression of kidney disease. The primary objective of the in-depth review was to answer the question, 'Do variations in dietary sodium consumption influence renal outcomes in patients with CKD prior to the development of ESRD?'

\section{Methods}

The in-depth review was conducted between March and November 2004 by a multidisciplinary team that included nephrologists and epidemiologists. The clinical question under study was operationally defined to include manipulation of salt consumption either higher (increase) or lower (decrease). Designated renal outcomes were defined as functional (e.g. changes in calculated or measured glomerular filtration rate, GFR), structural (e.g. renal scarring on imaging scans), surrogate (e.g. crystal depots excluding kidney stone formation), and/or pathologic (e.g. proteinuria, death). The renal outcome could be either acute (i.e. transient changes in renal function) or chronic (i.e. non-reversible changes).

\section{Data Sources and Search Strategy}

A comprehensive search strategy guided the survey for pertinent articles that addressed the study question. The search strategy included exploration of 7 electronic databases, a manual search of appropriate literature, and consultations with experts in the field. Articles were limited to human studies published as peer-reviewed journal articles, research reports or monographs, conference proceedings or abstracts, or dissertations published between January 1, 1966, and August 31, 2004. All electronic databases were searched using a basic search strategy that involved three components: defining the research questions into two components, salt consumption and kidney outcome; identification of standard Medical Subject Headings ( $\mathrm{MeSH}$ ) and related key terms and their variations for each of the two components of the research questions, and application of Boolean operators. The basic search strategy used the following terms for salt consumption (dietary sodium, salt intake) and kidney outcome (proteinuria, albuminuria, glomerular filtration rate, and kidney disease). When appropriate, minor modifications to the basic search template were made to optimize the search strategy in individual electronic databases. The 7 databases included: Ovid MEDLINE, EMBASE, Evidence Based Medicine Reviews (EBMR; includes ACP Journal Club [ACP], Cochrane Controlled Trials Register [CCTR], Cochrane Database of Systematic Reviews [COCH], and Database of Abstracts of Reviews of Effectiveness [DARE]), Cochrane Central Registry of Controlled Trials (CENTRAL), ProQuest, Current Controlled Trials, and Science Citation Index. The manual search encompassed identifying references cited in selected studies and references from clinical researchers in the field of nephrology.

\section{Study Selection}

This review included experimental and non-experimental study designs. Inclusion criteria were articles published, in any language, between January 1, 1966, and August 31,2004, as a peerreviewed journal article, research report or monograph, conference proceeding or abstract, or dissertation, and that involved adult ( $\geq 18$ years of age) human subjects that pertained to CKD (stages I-IV), and that specified variation in salt consumption. Exclusion criteria were articles inconsistent with the inclusion criteria, anecdotal, speculative or editorial in nature, and studies conducted on animals, pediatric ( $\leq 18$ years of age) participants, participants with ESRD undergoing dialysis or renal transplantation, and studies with either kidney stone formation or hypertension as the only renal outcome measured.

\section{Data Extraction}

Review of published data extraction guidelines included the Centre for Review and Dissemination recommendations [4] and prior research [5-8]. After the review, the two instruments developed and validated by Cho and Bero [8] which measure methodologic and non-methodologic quality were modified for the purposes of this review and utilized in this systematic review. Modifications included adding three items each to measure intervention/exposure and disease progression, excluding an item on case studies, and re-wording three questions. Methodologic quality of a study is defined as minimization of systematic bias and consistency of conclusions with results [8], and is determined by the extent to which authors explicitly describe the study design and analytical methods used in their studies. These descriptions provide the basis for determining the occurrence of systematic bias and the consistency of the reported results. Except for the question on study design, all questions on the methodologic quality instrument were assigned responses (point values) of 'Yes' (2), 'Partial' (1), 'No' and 'Not Applicable' (0). Points for the study design question ranged between 1 and 5 points (ranging from 1 point for case reports or case studies to 5 points for randomized experimental designs). All items were given equal weight. The 
total points assigned to a study were divided by the total possible points to yield a fraction between 0 and 1, with a score of 1 indicating the highest methodologic quality. Studies with quality scores at or above the median were arbitrarily classified as high methodologic quality.

The 6-item non-methodologic quality instrument, unlike Cho and Bero's original 7-item scale, measured clinical relevance, generalizability, and ethics. Responses for these items were 'Yes' (2), 'Partial' (1), or 'No' (0). All items were weighed equally. The overall non-methodologic quality score was the total points awarded divided by the total possible points, with a score of 1 denoting highest non-methodologic quality. Studies with quality score at or above the median were arbitrarily classified as high non-methodologic quality.

\section{Quality Assessment of Studies}

To standardize the review and scoring procedures between reviewers, the authors developed and pilot tested a detailed and clear definition and coding instructions protocol. Four reviewers independently reviewed and scored articles selected for the study. The reviewers were blinded to the articles' authors and their institutional affiliation, title, and journal. Each article was randomly assigned to two reviewers. After reviewing all the assigned articles, scores were tabulated for the methodologic and non-methodologic quality scores. Disagreement between paired reviewers was resolved by consensus or, if the reviewers were unable to reconcile differences, the article was reviewed by two content experts and scored by consensus.

\section{Data Synthesis}

Methodologic and clinical heterogeneity of the studies precluded the pooling of studies and computing effect estimates for the intervention. Methodologic heterogeneity included differences between studies in study designs, assessment methods and measures for exposures, confounding factors, and effect-modifying variables measured and included in predictive models. Clinical heterogeneity between the studies was evident in the types, amounts, and dosages of the intervention/exposures, and baseline presentation characteristics of the study participants. In light of these constraints, the study undertook a detailed systematic qualitative review of the existent literature addressing the research question and qualitatively synthesized data from studies classified as high methodologic quality.

\section{Results}

\section{Identified Studies}

In all, 16 studies [9-24] met the inclusion/exclusion criteria, and were included in the systematic review. One article was in German and another in Japanese, each of these articles was translated into English for review by the authors. Common reasons for exclusion from this extensive review were: the lack of original data reported, review articles, inclusion of only healthy subjects, and/or use of non-renal outcome measures.

\section{Characteristics and Quality of Identified Studies}

The characteristics of the studies are summarized in table 1 . Most noteworthy was the heterogeneity in study design and study populations included in the studies as a whole. Twelve of the 16 studies included were experimental studies; 3 were randomized, cross-over trials; 5 were non-randomized comparative trials, and 4 were non-randomized cross-over trials. Eight studies either excluded diabetic patients or did not state the diabetic status of their patients. Of the 16 studies, 4 were restricted to only nephrotic patients, 9 included patients with different degrees of proteinuria (both nephrotic and sub-nephrotic) and 1 included patients with normal urinary protein excretion. Two studies randomly selected patients from an identified population with kidney disease while three studies randomly allocated patients to the exposure. Four studies provided only a partial description of the exposure in terms of its type, amount, and schedule. Fourteen study results were partially generalizable to the CKD population and 14 studies measured therapeutic outcomes that were somewhat clinically applicable. Seven studies did not explicitly mention obtaining approval from an Institutional Review Board for the conduct of the published research and 1 study (partial reporting of Institutional Review Board approval) mentioned that the patients provided informed consent.

The overall quality (methodologic and non-methodologic) scores for each study were highly variable. The overall methodologic quality of the studies was low (median 0.48 ), whereas the non-methodologic quality was higher (median 0.67). Six studies had both methodologic and non-methodologic quality scores at or above the median scores $[9,12,13,15,22,23]$. Two studies had non-methodologic quality scores at or above the median score, but the methodologic quality scores were lower than the median score $[16,20]$; and two studies had methodologic quality scores above the median score, but the non-methodologic quality scores were lower than the median score $[11,19]$. In terms of methodologic quality, most of the studies lacked detailed descriptions of their methods (i.e. sample size estimations, appropriateness and selection of patients, patient allocation to exposure, blinding of investigator and/or patient, potential confounders and their control, measurement bias, and measurement of attrition), exposure (i.e. type, amount, and schedule for administration, co-interventions, and dose-response relationships), and disease progression (i.e. reliable baseline assessment of disease state, similarity of sub-groups of patients in baseline disease state, and disease outcome). 
Table 1. Characteristics of studies reviewed $(n=16)$

\begin{tabular}{|c|c|c|}
\hline Characteristics & $\mathrm{n}$ & $\%$ \\
\hline \multicolumn{3}{|l|}{ Study design } \\
\hline Experimental, randomized, cross-over trial & 3 & 19 \\
\hline $\begin{array}{l}\text { Experimental, non-randomized, comparative trial, } \\
\text { no placebo }\end{array}$ & 5 & 31 \\
\hline Experimental, non-randomized, cross-over trial & 4 & 25 \\
\hline Non-experimental, cohort, prospective & 1 & 6 \\
\hline Non-experimental, cohort, retrospective & 1 & 6 \\
\hline Non-experimental, cross-sectional & 2 & 13 \\
\hline \multicolumn{3}{|l|}{ Presentation characteristics } \\
\hline \multicolumn{3}{|l|}{ Diabetic status } \\
\hline Only included diabetic patients & 5 & 31 \\
\hline Included diabetic and non-diabetic patients & 3 & 19 \\
\hline Excluded diabetic patients & 4 & 25 \\
\hline Diabetic status not stated & 4 & 25 \\
\hline \multicolumn{3}{|l|}{ Proteinuria status } \\
\hline Only included non-nephrotic proteinuria patients & 4 & 25 \\
\hline $\begin{array}{l}\text { Included non-nephrotic and nephrotic proteinuria } \\
\text { patients }\end{array}$ & 9 & 56 \\
\hline Excluded nephrotic patients & 1 & 6 \\
\hline Nephrotic proteinuria status not stated & 2 & 13 \\
\hline \multicolumn{3}{|l|}{ Sample size } \\
\hline$<19$ & 4 & 25 \\
\hline $20-49$ & 7 & 44 \\
\hline$\geq 50$ & 5 & 31 \\
\hline \multicolumn{3}{|l|}{ Random selection of study participants } \\
\hline Yes & 1 & 6 \\
\hline Partial & 1 & 6 \\
\hline No, not applicable, not stated & 14 & 88 \\
\hline \multicolumn{3}{|l|}{ Random allocation of study participants to exposure } \\
\hline Yes & 1 & 6 \\
\hline Partial & 2 & 13 \\
\hline No, not applicable, not stated & 13 & 81 \\
\hline \multicolumn{3}{|l|}{ Description of $\mathrm{Na}$ exposure (type, amount, schedule) } \\
\hline Complete & 12 & 75 \\
\hline Partial & 4 & 25 \\
\hline \multicolumn{3}{|c|}{ Participant's baseline disease state reliably assessed and validated } \\
\hline Yes & 9 & 56 \\
\hline Partial & 6 & 38 \\
\hline No, or not applicable & 1 & 6 \\
\hline \multicolumn{3}{|l|}{ Progression of disease process during study and exposure } \\
\hline Outcome was irreversible & 2 & 13 \\
\hline Outcome was reversible & 11 & 69 \\
\hline Outcome not clearly stated & 3 & 19 \\
\hline \multicolumn{3}{|c|}{ Results generalizable to entire chronic kidney disease population } \\
\hline Yes & 1 & 6 \\
\hline Partial & 13 & 81 \\
\hline No & 2 & 13 \\
\hline \multicolumn{3}{|l|}{ Therapeutic outcomes measured important } \\
\hline Yes & 3 & 19 \\
\hline Partial & 11 & 69 \\
\hline No & 2 & 13 \\
\hline \multicolumn{3}{|l|}{ Institutional Review Board approval explicitly reported } \\
\hline Yes & 8 & 50 \\
\hline Partial & 1 & 6 \\
\hline No & 7 & 44 \\
\hline
\end{tabular}

${ }^{1}$ Numbers do not always add up to 100 due to rounding.

\section{Summaries and Synthesis of Studies with High Methodologic Quality}

Although 16 studies met the inclusion-exclusion criteria, only 8 studies were found to have high methodologic quality scores [9,11-13, 15, 19, 22, 23]. Below are study summaries by design and a qualitative synthesis of data of these 8 studies.

Experimental, Randomized, Cross-Over Trials

Two of the 3 experimental, randomized, cross-over trials received high methodologic quality scores. The first study of 22 hypertensive patients found that modest changes in dietary salt intake (from 100 to $200 \mathrm{mEq} \mathrm{Na}^{+}$/ day) was associated with significant changes in proteinuria in salt-sensitive patients, but not in salt-resistant patients [23]. The second study included 41 type 2 diabetic, non-hypertensive patients with or without proteinuria matched on age, sex distribution, body mass index, duration of diabetes, and blood pressure. This study found that high salt intake increased the albumin excretion rate in microalbuminuric patients, but not in normoalbuminuric patients [22].

Experimental, Non-Randomized, Comparative Trials

Three of the 5 experimental, non-randomized, comparative trials received high methodologic quality scores. The first study among 81 male patients recruited from hospital admissions with uncomplicated, essential hypertension reported that black hypertensive patients had a significantly greater increase in GFR during a high salt diet than during a low salt diet; whereas, the GFR did not change in white hypertensive patients in response to changes in dietary sodium intake [19]. The order of dietary period was the same for all patients (high salt, then low salt). A second study examined renal adaptation to dietary sodium restriction among 7 healthy adult medical staff volunteers and 14 adult normotensive patients with primary glomerular disease (half of the patients had a moderate reduction in renal function and the other half had normal renal function) recruited from an outpatient clinic [11]. The study participants received a diet containing $35 \mathrm{mEq}$ of sodium and a supplement of $200 \mathrm{mEq}$ of sodium oral capsules for 7 days (normal sodium diet), after which the sodium supplement was withdrawn to achieve a low sodium diet state. Those with kidney disease had a significant decrease in GFR. The final study in this category reported retrospectively on 57 patients seen at an outpatient clinic [12]. The patients selected possessed creatinine clearance values between 10 and $40 \mathrm{ml} /$ min and dietary salt intakes of $<100$ or $>200 \mathrm{mEq} /$ day. 
Over the 3-year period of assessment, an improvement in renal outcome was observed in patients undergoing salt restriction.

Experimental, Non-Randomized, Cross-Over Trials

Three of the 4 experimental, non-randomized, crossover trials received high methodologic quality scores. The first study examined whether sodium sensitivity of blood pressure increased before hypertension begins and whether this sensitivity is related to albuminuria in a sample of 32 randomly selected, inpatients with type 2 diabetes [15]. After random selection, study participants were divided into 3 groups based on level of albuminuria on a regular diet ( $\sim 150 \mathrm{mmol}$ of sodium/day), and then dietary sodium was manipulated for 1 week each in random order and with no intervening periods between two different diets (low sodium $\sim 80 \mathrm{mmol} /$ day or ordinary sodium $\sim 200 \mathrm{mmol} /$ day). The salt sensitivity index, which shows the sodium sensitivity of blood pressure independent of the magnitude of the change in sodium intake and is the reciprocal of slope of the pressurenatriuresis curve, was higher among the micro- and macroalbuminuric patients than the normoalbuminuric patients.

The second study, conducted among 15 adult patients with nephropathy from type 2 diabetes, assessed whether sodium intake altered albumin excretion when the patients were treated with two different long-acting calcium antagonists [9]. Over four 4-week periods, all patients received the following dietary sodium intake and drug, with time periods noted parenthetically: $50 \mathrm{mEq}$ of sodium/day ( 2 weeks), $50 \mathrm{mEq}$ of sodium/day and clonidine (4 weeks), $50 \mathrm{mEq}$ of sodium/day and nifedipine (4 weeks), $250 \mathrm{mEq}$ of sodium/day and nifedipine (4 weeks), and $250 \mathrm{mEq}$ of sodium/day and clonidine ( 4 weeks). The protocol was then repeated with diltiazem. The study reported no reduction in albumin excretion when patients received a diet of $250 \mathrm{mEq}$ of sodium/ day, regardless of the antihypertensive medication used. A diet of $50 \mathrm{mEq}$ of sodium/day and diltiazem (but not nifedipine) reduced the excretion of albumin.

A third study, conducted among patients with nonsteroid sensitive proteinuria $(>3 \mathrm{~g} / 24 \mathrm{~h}$ ) and a GFR of $>30 \mathrm{ml} / \mathrm{min} / 1.73 \mathrm{~m}^{2}$, determined the efficacy of the ACE inhibitor lisinopril in treating overt proteinuria in comparison with the NSAID indomethacin [13]. The exposure (dietary salt) was varied for 9 of the 12 patients recruited into the study. These patients received lisinopril along with a low sodium diet ( $50 \mathrm{mmol} / \mathrm{day})$, followed by a high sodium diet $(200 \mathrm{mmol} /$ day $)$, and again followed by a low sodium diet. The study reported a significant decrease in proteinuria when patients changed from a high sodium to a low sodium diet during lisinopril therapy.

The majority of the studies (6 of 8) reported an association between dietary sodium intake and kidney function. Two studies $[22,23]$ reported a direct (positive) correlation between $\mathrm{Na}^{+}$intake and urinary protein excretion. Two additional studies $[13,19]$ showed a direct relationship between $\mathrm{Na}^{+}$intake and urinary protein excretion in study subgroups (one in African-Americans, the other with the use of ACE inhibitors). One study [23] showed an acute (short-term) increase in GFR with increased $\mathrm{Na}^{+}$intake, while 2 other studies $[11,12]$ showed a slowing in the rate of decline in renal function as estimated by the reciprocal of creatinine over time. Two studies $[9,15]$ did not have sufficient information to comment on the influence of dietary sodium on renal outcomes.

All of the 8 studies summarized have several common factors that limit the robustness of the clinical findings, including: (1) brief follow-up periods of days to weeks to examine the association between variations in dietary salt intake and kidney disease, except one study [6] which used retrospective data for a 3-year period; (2) small sample sizes for the studies (ranging from 12 to 41 participants) especially for comparative analyses with two exceptions [12, 19]; (3) no reports of concealment of allocation with two exceptions [11,22], and (4) only one study [23] reported on random selection of their study participants, and this study did not describe their random selection procedures.

\section{Discussion}

The findings of this in-depth review, which examined the relationship between dietary salt and progression of renal disease, are based on a diverse group of studies with a variety of methodologies, study populations and targeted outcomes. This malignant heterogeneity limits our ability to make strong inferences that will impact clinical practice; however, in this collection of studies there is scientific merit that increased dietary salt exposure is toxic to the kidneys.

Five studies $[9,13,15,19,20]$ displayed results indicating that increased dietary salt resulted in increased blood pressure, albuminuria or filtration fraction; one suggested this was more common in African-Americans compared to Caucasians [19], and another suggested this was 
more common in individuals prescribed diuretics [10]. Increased salt sensitivity of blood pressure was correlated with increased albuminuria [15] in another study. There were three additional studies [11, 12, 16], which demonstrated that reduced dietary salt was associated with diminished albuminuria and/or diminished likelihood for progressive reduction of estimated GFR. Only two studies $[17,18]$ suggested that reduced sodium diet was not beneficial in slowing loss of kidney function, and these studies received the lowest methodologic quality scores in our review.

Due to the overall poor methodologic quality, the available studies provide insufficient data to answer our primary question and support our hypothesis that increased salt intake is nephrotoxic. Despite the limited evidence, it is plausible that dietary salt can both initiate and propagate renal injury independent of raising blood pressure [25]. In several experimental trials involving animal models, a direct relationship between greater salt intake and increased fibrosis mediators, mainly transforming growth factor- $\beta$ (TGF- $\beta$ ), and reactive oxidative stressors [25-39] has been demonstrated. These substances have been linked to both tissue and vascular injury [25].

The data linking dietary salt and stimulation of TGF- $\beta$ are consistent whether one looks at either in vitro [39] or in vivo [38] experimental models. Increased sodium chloride in the solution bathing isolated aortic rings results in increased TGF- $\beta$ production [39]. This effect is diminished after removing the endothelium suggesting that this may be the target of the increased salt exposure. Similarly, increased dietary salt intake in Sprague-Dawley rats results in increased renal cortical concentration of TGF- $\beta$ [39]. The relationship between salt exposure and vascular production of TGF- $\beta$ could result not only in impaired vascular function, but also remodeling and restructuring.

Additional experimental studies [27, 31-37] have demonstrated the development of an unfavorable balance of reactive oxygen species with increased dietary salt exposure in salt-sensitive rat models of hypertension. The combination of increased reactive oxygen species and enhanced TGF- $\beta$ production could also lead to vascular dysfunction, higher levels of blood pressure, and greater proclivity for vascular injury. The well-described relationship between dietary salt and increased blood pressure in some patients may, in fact, be related to alterations in TGF- $\beta$ levels and increased oxidative stress.

Our in-depth review was limited by uniform methodologic flaws observed in the studies, including inconsistent terminology, no report of inclusion and/or exclusion criteria, lack of random allocation, lack of randomization, lack of masking, and small sample sizes. Additionally, the heterogeneity and serious methodologic flaws limited our ability to perform a meta-analysis. Although we were limited in our statistical analysis, this is the only in-depth review to evaluate the evidence about the impact of salt on renal disease, independent of blood pressure. Our multidisciplinary team of nephrologists and epidemiologists used a comprehensive search strategy, including online and manual searches, and correspondence with experts worldwide to minimize publication bias, and we used a validated data capture tool in an effort to standardize our approach and derive composite conclusions based on common observations. Finally, all reviewers were blinded to author(s) and journal(s), minimizing bias.

Even though we have noted the heterogeneity of the existing literature, there is substantial consistency in the conclusion that there is an association between salt consumption and clinical indicators of renal injury, especially proteinuria. Our systematic review suggests that dietary salt may be an overlooked, modifiable exposure for CKD. These observations are supported by experimental data linking salt exposure with increased TGF- $\beta$ levels and reactive oxygen species. For the present, there is insufficient information to change recommendations with regard to diet and its constituents. However, since there was no evidence of a detrimental effect of reduced salt intake, and often patients with CKD have blood pressure salt sensitivity, it would be prudent to consider modifying dietary salt intake until better data becomes available. This review illustrates the challenges that future trials in this area will have to overcome in order to improve upon study quality. For example, one methodologic hurdle noted in the available literature results from the utilization of urinary electrolyte excretion as a measure of ingestion, an approach that needs to be validated in light of the complex interaction between the kidney and salt metabolism. Additionally, these studies used surrogate measures for progression of kidney disease, such as a change in reciprocal slope of creatinine or increases in urinary albumin excretion, which may have only limited ability to predict ESRD. Moreover, many of the studies utilized experimental designs where there were manipulations in salt ingestion with measured changes observed over short intervals. To what extent such observations can be translated into conclusions about harm from long-term excessive salt consumption and risk of ESRD is unknown.

Clinical trials are needed to more carefully examine dietary salt consumption patterns utilizing simple measures, which could be validated and utilized on a large 
scale in clinical practice. There are several ongoing, prospective cohort trials enrolling CKD patients (e.g. CRIC - The Chronic Renal Insufficiency Cohort Study; FAVORIT - The Folic Acid for Vascular Outcome in Transplantation, etc.) that could serve as vehicles for a properly designed ancillary study to evaluate the association between salt consumption and clinical indicators of renal injury. The ultimate goal of such an ancillary study is to develop clinical guidelines for CKD patients. In the meantime, modest dietary avoidance of salt should be encouraged in CKD patients, especially if they have hypertension and/or proteinuria.

\section{Acknowledgments}

This research was supported by a grant from the National Center for Minority Health and Health Disparities (P60MD000532). The contents of the article are solely the responsibilities of the authors and do not necessarily represent the views of the funding agency. The authors acknowledge the invaluable expertise and guidance provided by Paula G. Raimondo, MLS, AHIP, from the Health Sciences and Human Services Library, University of Maryland, School of Medicine, towards the development and implementation of the literature searches and Drs Surjo Soekadar and Tsuyoshi Mitarai for translating the non-English language articles.

\section{References}

1 Jafar TH, Schmid CH, Landa M, et al: Angiotensin-converting enzyme inhibitors and progression of nondiabetic renal disease. A meta-analysis of patient-level data. Ann Intern Med 2001;135:73-87.

-2 Swift PA, Markandu ND, Sagnella GA, He FJ, MacGregor GA: Modest salt reduction reduces blood pressure and urine protein excretion in black hypertensives: a randomized control trial. Hypertension 2005;46:308312.

$>$ Weir MR, Fink JC: Salt intake and progression of chronic kidney disease: an overlooked modifiable exposure? A commentary. Am J Kidney Dis 2005;45:176-188.

4 NHS Centre for Reviews and Dissemination: Undertaking Systematic Reviews of Research on Effectiveness. CRD's Guidance for Those Carrying Out or Commissioning Reviews. CRD Report Number 4, ed 2. York, University of York 2001.

$\checkmark 5$ Juni P, Witschi A, Bloch R, Egger M: The hazards of scoring the quality of clinical trials for meta-analysis. JAMA 1999;282:10541060.

6 Juni P, Altman DG, Egger M: Systematic reviews in health care: assessing the quality of controlled clinical trials. BMJ 2001;323:4246.

7 Deeks JJ: Systematic reviews in health care: systematic reviews of evaluations of diagnostic and screening tests. BMJ 2001;323: 157-162.

$\checkmark 8$ Cho MK, Bero LA: Instruments for assessing the quality of drug studies published in the medical literature. JAMA 1994;272:101104.

9 Bakris GL, Smith A: Effects of sodium intake on albumin excretion in patients with diabetic nephropathy treated with long-acting calcium antagonists. Ann Intern Med 1996; 125:201-204.
0 Buter H, Hemmelder MH, Navis G, de Jong PE, de Zeeuw D: The blunting of the antiproteinuric efficacy of ACE inhibition by high sodium intake can be restored by hydrochlorothiazide. Nephrol Dial Transplant 1998; 13:1682-1685.

11 Cianciaruso B, Bellizzi V, Minutolo R, et al: Renal adaptation to dietary sodium restriction in moderate renal failure resulting from chronic glomerular disease. J Am Soc Nephrol 1996;7:306-313.

12 Cianciaruso B, Bellizzi V, Minutolo R, et al: Salt intake and renal outcome in patients with progressive renal disease. Miner Electrolyte Metab 1998;24:296-301.

13 Heeg JE, de Jong PE, van der Hem GK, de Zeeuw D: Efficacy and variability of the antiproteinuric effect of ACE inhibition by lisinopril. Kidney Int 1989;36:272-279.

14 Holler C, Abrahamian H, Auinger M: Effect of nutrition on microalbuminuria in patients with type 1 diabetes: prospective data evaluation over 5 years (in German). Acta Med Austriaca 1999;26:168-172.

15 Imanishi M, Yoshioka K, Okumura M, et al: Sodium sensitivity related to albuminuria appearing before hypertension in type 2 diabetic patients. Diabetes Care 2001;24:111116.

16 Kuriyama S, Tomonari H, Ohtsuka Y, Yamagishi $\mathrm{H}$, Ohkido I, Hosoya T: Salt intake and the progression of chronic renal diseases (in Japanese). Nippon Jinzo Gakkai Shi 2003;45: 751-758.

17 Mazouz H, Kacso I, Ghazali A, et al: Risk factors of renal failure progression two years prior to dialysis. Clin Nephrol 1999;51:355366.

18 Metcalf PA, Baker JR, Scragg RK, Dryson E, Scott AJ, Wild CJ: Dietary nutrient intakes and slight albuminuria in people at least 40 years old. Clin Chem 1993;39:2191-2198.
19 Parmer RJ, Stone RA, Cervenka JH: Renal hemodynamics in essential hypertension. Racial differences in response to changes in dietary sodium. Hypertension 1994;24:752757.

20 Schiffl H, Kuchle C, Lang S: Dietary salt, intracellular ion homeostasis and hypertension secondary to early-stage kidney disease. Miner Electrolyte Metab 1996;22:178-181.

21 Shiigai T, Shichiri M: Late escape from the antiproteinuric effect of ace inhibitors in nondiabetic renal disease. Am J Kidney Dis 2001;37:477-483.

22 Vedovato M, Lepore G, Coracina A, et al: Effect of sodium intake on blood pressure and albuminuria in type 2 diabetic patients: the role of insulin resistance. Diabetologia 2004; 47:300-303.

23 Weir MR, Dengel DR, Behrens MT, Goldberg AP: Salt-induced increases in systolic blood pressure affect renal hemodynamics and proteinuria. Hypertension 1995;25: 1339-1344.

24 Yoshioka K, Imanishi M, Konishi Y, et al: Glomerular charge and size selectivity assessed by changes in salt intake in type 2 diabetic patients. Diabetes Care 1998;21:482486.

25 Mishra SI, Jones-Burton C, Fink JC, Brown J, Bakris GL, Weir MR: Does dietary salt increase the risk for progression of kidney disease? Curr Hypertens Rep 2005;7:385-391.

26 Barton M, Vos I, Shaw S, et al: Dysfunctional renal nitric oxide synthase as a determinant of salt-sensitive hypertension: mechanisms of renal artery endothelial dysfunction and role of endothelin for vascular hypertrophy and glomerulosclerosis. J Am Soc Nephrol 2000;11:835-845.

27 Boegehold MA: Effect of dietary salt on arteriolar nitric oxide in striated muscle of normotensive rats. Am J Physiol 1993;264: H1810-H1816. 
28 Chen PY, Sanders PW: L-Arginine abrogates salt-sensitive hypertension in Dahl/Rapp rats. J Clin Invest 1991;88:1559-1567.

29 Frisbee JC, Lombard JH: Chronic elevations in salt intake and reduced renal mass hypertension compromise mechanisms of arteriolar dilation. Microvasc Res 1998;56:218227.

-30 Frisbee JC, Lombard JH: Acute elevations in salt intake and reduced renal mass hypertension compromise arteriolar dilation in rat cremaster muscle. Microvasc Res 1999;57: 273-283.

-31 Hannken T, Schroeder R, Stahl RA, Wolf G: Angiotensin II-mediated expression of p27Kip1 and induction of cellular hypertrophy in renal tubular cells depend on the generation of oxygen radicals. Kidney Int 1998; 54:1923-1933.
32 Inoue N, Venema RC, Sayegh HS, Ohara Y, Murphy TJ, Harrison DG: Molecular regulation of the bovine endothelial cell nitric oxide synthase by transforming growth factorbeta 1. Arterioscler Thromb Vasc Biol 1995; 15:1255-1261.

33 Kitiyakara C, Chabrashvili T, Chen Y, et al: Salt intake, oxidative stress, and renal expression of NADPH oxidase and superoxide dismutase. J Am Soc Nephrol 2003;14:27752782.

34 Lenda DM, Sauls BA, Boegehold MA: Reactive oxygen species may contribute to reduced endothelium-dependent dilation in rats fed high salt. Am J Physiol Heart Circ Physiol 2000;279:H7-H14.

35 Lenda DM, Boegehold MA: Effect of a highsalt diet on oxidant enzyme activity in skeletal muscle microcirculation. Am J Physiol Heart Circ Physiol 2002;282:H395-H402.
36 Nicod L, Rodriguez S, Letang JM, et al: Antioxidant status, lipid peroxidation, mixed function oxidase and UDP-glucuronyl transferase activities in livers from control and DOCA-salt hypertensive male Sprague Dawley rats. Mol Cell Biochem 2000;203:33-39.

37 Schultz PJ, Tolins JP: Adaptation to increased dietary salt intake in the rat: role of endogenous nitric oxide. J Clin Invest 1993;91:642650.

38 Ying WZ, Sanders PW: Dietary salt modulates renal production of transforming growth factor-beta in rats. Am J Physiol 1998;274:F635-F641.

39 Ying WZ, Sanders PW: Dietary salt increases endothelial nitric oxide synthase and TGFbeta1 in rat aortic endothelium. Am J Physiol 1999;277:H1293-H1298. 